\section{Exames de apoio ao diagnóstico}

Pacientes com TEP apresentam-se frequentemente com sintomas torácicos agudos. Nesse sentido, existem exames que auxiliam no diagnóstico diferencial, reforçando a suspeita clínica ou tendendo a outro diagnóstico, mas não permitindo confirmar ou excluir com segurança o diagnóstico de TEP. Exames séricos, como leucograma, enzimas cardíacas, gasometria arterial e provas hepáticas, demonstraram alterações em percentuais variáveis dependendo da série estudada e da gravidade da apresentação de TEP. Entre eles, a gasometria arterial é a mais estudada. Apesar de possível, é incomum TEP com valores gasométricos $\left(\mathrm{PaO}_{2}, \mathrm{PaCO}_{2}\right.$ e gradiente alvéolo-arterial) inteiramente normais.

\section{Radiografia de tórax}

Os achados à radiografia de tórax raramente são conclusivos para o diagnóstico de TEP aguda, sendo mais importantes para o diagnóstico diferencial e para a avaliação da cintilografia de perfusão. Pode ser normal e, na presença de dispneia de causa não definida, reforça a suspeita de TEP aguda. Os achados mais comuns são atelectasias laminares nas bases, elevação da cúpula diafragmática e derrame pleural, geralmente pequeno. Podem-se observar sinais clássicos de oligoemia regional, aumento das artérias pulmonares centrais e opacidade periférica em cunha. ${ }^{(1-3)}$

A radiografia de tórax é útil para o diagnóstico diferencial de TEP, permitindo somente evidenciar outras causas para os sintomas, mas não excluir ou confirmar TEP.(C)

\section{ECG}

É incomum o ECG ser normal, e é infrequente o achado do padrão clássico S1-Q3-T3 descrito para TEP aguda. Às vezes, a única alteração é a presença de taquicardia sinusal. Há outros sinais de sobrecarga direita que podem ser observados, além do padrão S1-Q3-T3, principalmente em pacientes com TEP maciça, como desvio do eixo QRS para a direita, inversão de onda T nas precordiais de $\mathrm{V} 1-\mathrm{V} 3$, bloqueio do ramo direito transitório total ou parcial, padrão Qr em V1, onda $\mathrm{P}$ pulmonale, onda $\mathrm{Q}$ na derivação 111 , padrão Q3-T3 e taquiarritmias atriais. ${ }^{(2-4)}$ Todos os achados são inespecíficos, e a principal relevância do exame é excluir outras entidades, como 1AM ou pericardite. ${ }^{(2)}$ Alguns estudos têm avaliado o ECG para a estratificação de risco, o diagnóstico e o prognóstico, mas demonstraram pouca utilidade clínica devido à baixa sensibilidade e à baixa razão de probabilidade. ${ }^{(4-6)}$

0 ECG, à semelhança da radiografia de tórax, é útil, sobretudo, para o diagnóstico diferencial de TEP.(C)

\section{Ecocardiograma}

0 ecocardiograma é um exame não invasivo e de baixa sensibilidade para o diagnóstico de TEP. Entretanto, pode ser uma ferramenta útil no diferencial de dispneia aguda, dor torácica, colapso cardiovascular e outras situações clínicas em que a TEP é considerada como um dos diagnósticos. ${ }^{(3)}$ Outra importância do ecocardiograma é na avaliação prognóstica e na estratificação de risco de pacientes com TEP. . $^{(7,8)}$

0 ecocardiograma transtorácico raramente consegue visualizar o êmbolo pulmonar, mas às vezes revela um trombo flutuando no átrio ou no VD. É incapaz de excluir TEP com segurança, e sua principal relevância é em pacientes hemodinamicamente instáveis com TEP maciça, nos quais a visualização do trombo proximal (que confirma o diagnóstico, em geral, pelo ecocardiograma transesofágico), a dilatação aguda do VD e a presença de hipertensão pulmonar (diagnóstico presuntivo) permitirão a rápida decisão da terapêutica (uso de trombolíticos). ${ }^{(9-12)}$ Embolia pulmonar com importante repercussão hemodinâmica é improvável na presença de ecocardiograma normal. 0 ecocardiograma transesofágico à beira do leito pode ser considerado como o teste diagnóstico de primeira escolha, podendo confirmar embolia pulmonar em pacientes em choque ou durante a ressuscitação cardiopulmonar. ${ }^{(3,13)}$

0 ecocardiograma pode ser útil no diagnóstico de TEP clinicamente maciça.(C)

\section{Dímero $D$}

0 dímero $\mathrm{D}$ é um produto da degradação da fibrina, podendo estar elevado na presença de trombos, mas também em outras situações, como no pós-operatório, na gestação, no puerpério, na doença vascular periférica, no câncer, na 
insuficiência renal, na sepse e em várias doenças inflamatórias, assim como aumenta com a idade, o que limita sua utilidade clínica. ${ }^{(3,14)} \mathrm{Tem}$ alta sensibilidade, mas a sua especificidade é baixa; portanto, deve ser analisado com cautela, em conjunto com a avaliação de probabilidade clínica. ${ }^{(15)}$

Há diferentes métodos para a realização do teste (ELISA ou ELISA rápido quantitativo, semiquantitativo ou qualitativo; aglutinação por látex quantitativo ou semiquantitativo ou aglutinação do sangue total), diferindo, entre eles, na sensibilidade, especificidade e razão de probabilidade, além da variabilidade entre pacientes com suspeita de TVP ou TEP. Dentre todos os métodos utilizados, o teste por ELISA ou ELISA rápido quantitativo são os que têm melhor sensibilidade e maior probabilidade de excluir TEP (razão de probabilidade negativa) e são os que têm melhor utilidade clínica. 0 dímero $\mathrm{D}$ é um teste unidirecional; logo, um teste negativo é usado para excluir o diagnóstico. ${ }^{(1,16)}$

Numa revisão sistemática para a avaliação de estratégias para o diagnóstico de TEP aguda em pacientes com baixa probabilidade clínica préteste, um teste negativo para dímero D (qualquer teste) esteve associado com uma probabilidade pós-teste $<5 \%$, não sendo necessários outros testes para se excluir a embolia pulmonar. ${ }^{(1,12)}$ Já em pacientes com probabilidade clínica intermediária, para a exclusão de TEP, só pode ser valorizado um teste quantitativo utilizando o dímero D pelo método ELISA (valor < $500 \mu \mathrm{g} / \mathrm{L}$ ). Em pacientes com alta probabilidade clínica, outros testes serão necessários para se excluir esse diagnóstico com segurança. Nesses pacientes, se o teste for positivo, não acrescentará ajuda no diagnóstico, sendo que outros exames deverão ser realizados. ${ }^{(15)}$

Recomenda-se o seguinte:

- 0 dímero D deve ser usado somente em pacientes após a avaliação da probabilidade clínica.(B)

- 0 dímero $\mathrm{D}$ não deve ser usado em pacientes com alta probabilidade clínica. (B)

- Um teste negativo exclui TEP em pacientes com baixa (qualquer método) ou intermediária (ELISA) probabilidade clínica, sem a necessidade de exames de imagem adicionais.(B)

\section{Referências}

1. Langan CJ, Weingart S. New diagnostic and treatment modalities for pulmonary embolism: one path through the confusion. Mt Sinai J Med. 2006;73(2):528-41.

2. Riedel M. Diagnosing pulmonary embolism. Postgrad Med J. 2004;80(944):309-19.

3. Guidelines on diagnosis and management of acute pulmonary embolism. Task Force on Pulmonary Embolism, European Society of Cardiology. Eur Heart J. 2000;21(16):1301-36.

4. Sinha N, Yalamanchili K, Sukhija R, Aronow WS, Fleisher AG, Maguire GP, et al. Role of the 12-lead electrocardiogram in diagnosing pulmonary embolism. Cardiol Rev. 2005;13(1):46-9.

5. lles S, Le Heron CJ, Davies G, Turner JG, Beckert LE. ECG score predicts those with the greatest percentage of perfusion defects due to acute pulmonary thromboembolic disease. Chest. 2004;125(5):1651-6.

6. Geibel A, Zehender M, Kasper W, Olschewski M, Klima C, Konstantinides SV. Prognostic value of the ECG on admission in patients with acute major pulmonary embolism. Eur Respir J. 2005;25(5):843-8.

7. Kucher N, Rossi E, De Rosa M, Goldhaber SZ. Prognostic role of echocardiography among patients with acute pulmonary embolism and a systolic arterial pressure of $90 \mathrm{~mm} \mathrm{Hg}$ or higher. Arch Intern Med. 2005;165(15):1777-81.

8. Vieillard-Baron A, Page B, Augarde R, Prin S, Qanadli $\mathrm{S}$, Beauchet $\mathrm{A}$, et al. Acute cor pulmonale in massive pulmonary embolism: incidence, echocardiographic pattern, clinical implications and recovery rate. Intensive Care Med. 2001;27(9):1481-6.

9. Bova C, Greco F, Misuraca G, Serafini 0, Crocco F, Greco A, et al. Diagnostic utility of echocardiography in patients with suspected pulmonary embolism. Am J Emerg Med. 2003;21(3):180-3.

10. Miniati M, Monti S, Pratali L, Di Ricco G, Marini C, Formichi B, et al. Value of transthoracic echocardiography in the diagnosis of pulmonary embolism: results of a prospective study in unselected patients. Am J Med. 2001;110(7):528-35.

11. Pruszczyk P, Torbicki A, Kuch-Wocial A, Szulc M, Pacho R. Diagnostic value of transoesophageal echocardiography in suspected haemodynamically significant pulmonary embolism. Heart. 2001;85(6):628-34.

12. Roy PM, Colombet 1, Durieux P, Chatellier G, Sors H, Meyer G. Systematic review and meta-analysis of strategies for the diagnosis of suspected pulmonary embolism. BMJ. 2005;331(7511):259.

13. Vieillard-Baron A, Prin S, Chergui K, Dubourg 0, Jardin F. Echo-Doppler demonstration of acute cor pulmonale at the bedside in the medical intensive care unit. Am J Respir Crit Care Med. 2002;166(10):1310-9.

14. Wells PS. Advances in the diagnosis of venous thromboembolism. J Thromb Thrombolysis. 2006;21(1):31-40.

15. British Thoracic Society Standards of Care Committee Pulmonary Embolism Guideline Development Group. British Thoracic Society guidelines for the management of suspected acute pulmonary embolism. Thorax. 2003;58(6):470-83.

16. Stein PD, Hull RD, Patel KC, Olson RE, Ghali WA, Brant $R$, et al. D-dimer for the exclusion of acute venous thrombosis and pulmonary embolism: a systematic review. Ann Intern Med. 2004;140(8):589-602. 